\title{
Clinical and pathological benefit of Twendee $X$ in Alzheimer's disease transgenic mice with chronic cerebral hypoperfusion
}

Xia Liu, Toru Yamashita, Jingwei Shang, Xiaowen Shi, Ryuta Morihara, Yong Huang, Kota Sato, Mami Takemoto, Nozomi Hishikawa, Yasuyuki Ohta, and Koji Abe

Department of Neurology, Graduate School of Medicine, Dentistry and Pharmaceutical Sciences, Okayama University, 2-5-1 Shikatacho, Kitaku, Okayama 700-8558, Japan

Running headline: Neuroprotective effect of Twendee X.

Keywords: Alzheimer's disease, APP23 mice, chronic cerebral hypoperfusion, antioxidative, anti-inflammatory.

Corresponding author: Prof. Koji Abe, Department of Neurology, Okayama University Graduate School of Medicine, Dentistry and Pharmaceutical Sciences, 2-5-1 Shikata-cho, Okayama 700-8558, Japan. E-mail: pggx277x@hotmail.com

\footnotetext{
Abbreviations used: ameriod constrictors (ACs); Alzheimer's disease (AD); amyloid- $\beta$ (A $\beta$ ); bilateral common carotid arteries (BCCAs); bovine serum albumin (BSA); cerebral blood flow (CBF); chronic cerebral hypoperfusion $(\mathrm{CCH})$; cortex (CTX); dentate gyrus (DG); hippocampus (HI); months (M); phosphate-buffered saline (PBS); paraformaldehyde (PFA); subiculum (Sub); thalamus (TH); Twendee X (TwX); wild type (WT).
} 


\begin{abstract}
Background: Multiple pathogeneses are involved in Alzheimer's disease, such as amyloid- $\beta$ accumulation, neuroinflammation and oxidative stress. The pathological impact of chronic cerebral hypoperfusion on Alzheimer's disease is still poorly understood.
\end{abstract}

Methods: APP23 mice were implanted to bilateral common carotid arteries stenosis with ameroid constrictors for slowly progressive chronic cerebral hypoperfusion. The effects of the administration of Twendee $\mathrm{X}$ were evaluated by behavioral analysis, immunohistochemical analysis and immunofluorescent histochemistry.

Results: In the present study, chronic cerebral hypoperfusion, which is commonly found in aged Alzheimer's disease, significantly exacerbated motor dysfunction of APP23 mice from 5 months and cognitive deficit from 8 months of age, as well as neuronal loss, extracellular amyloid- $\beta$ plaque and intracellular oligomer formations, and amyloid angiopathy at 12 months. Severe upregulations of oxidative markers and inflammatory markers were found in the cerebral cortex, hippocampus and thalamus at 12 months. Twendee $X$ treatment $(20 \mathrm{mg} / \mathrm{kg} / \mathrm{d}$, from 4.5 to 12 months) substantially rescued the cognitive deficit and reduced the above amyloid- $\beta$ pathology and neuronal loss, alleviated neuroinflammation and oxidative stress.

Conclusions: The present findings suggested a potential therapeutic benefit of Twendee X for Alzheimer's disease with chronic cerebral hypoperfusion. 


\section{Introduction}

Alzheimer's disease (AD) is the most common cause of dementia, accounting for $69 \%$ in all dementia among the people older than 75 years. ${ }^{1}$ Although pathogenesis of $\mathrm{AD}$ is complex, ${ }^{2-3}$ oxidative stress and inflammation are also considered to play important roles in the process of $\mathrm{AD} .{ }^{4-5}$ Chronic cerebral hypoperfusion $(\mathrm{CCH})$ is a major cause of cognitive deficits and contributes to the progression of dementia. ${ }^{6}$ Our recent studies showed that $\mathrm{CCH}$ strongly enhanced the AD pathology in mice, ${ }^{7-8}$ which is appropriate to study the effects of $\mathrm{CCH}$ on cognitive impairments, AD pathology, neuroinflammatory and oxidative stress. Twendee $\mathrm{X}$ (TwX) is an anti-oxidant mixture that contains multiple ingredients, such as coenzyme Q10, ascorbic acid, L-glutamine and cystine. Our previous study demonstrated neuroprotective effects of TwX in an acute cerebral ischemia model in mice by reducing ischemic infarct and attenuating both oxidative stress and inflammatory markers. ${ }^{9}$

In the present study, therefore, we investigated a possible therapeutic effect of TwX on cognitive function, $\mathrm{A} \beta$ pathology, inflammatory and oxidative stresses in an $\mathrm{AD}$ mouse model with $\mathrm{CCH}$.

\section{Materials and Methods}

\section{Mouse model}

All procedures were approved by the guidelines of the Animal Committee of the Graduate School of Medicine and Dentistry of Okayama University (OKU-2014-095). APP23 mice overexpress human APP751 isoform carrying the Swedish double mutation (KM670/671NL) under the control of the murine Thy1 promoter. ${ }^{10}$ APP23 male mice were obtained from Dr. Takashi Saito (RIKEN Brain Science Institute, Saitama, Japan) and maintained as hemizygotes 
by mating APP23 male mice with C57BL/6J female mice (CLEA Japan, Tokyo, Japan). The offspring were genotyped using a PCR assay with DNA obtained from tail tissue samples. Wild type (non-transgenic) littermates were used as controls. Mice were housed in 12:12-hour lightdark cycle with controlled temperature and free access to food and water.

Four groups of mice were used in this study: wild type mice (WT + sham surgery, $\mathrm{n}=10$ ), APP23 group (APP23 + sham surgery, $\mathrm{n}=10), \mathrm{CCH}$ group $(\mathrm{APP} 23+\mathrm{CCH}, \mathrm{n}=12)$ and $\mathrm{TwX}$ group (APP23 $+\mathrm{CCH}+\mathrm{TwX}, \mathrm{n}=13)$. Groups comprised approximately equal numbers of male and female mice.

For the $\mathrm{CCH}$ and $\mathrm{TwX}$ mice, ameriod constrictors (ACs) were applied to the bilateral common carotid arteries (BCCAs) at 4 months $(\mathrm{M})$ of age, and cerebral blood flow (CBF) was measured with a laser-Doppler flowmeter (FLO-C1, Omegawave, Tokyo, Japan) before and 1, $3,7,14$ and $28 \mathrm{~d}$ after surgery as our previously report. ${ }^{8}$

TwX is a mixture containing multiple antioxidants, ${ }^{9}$ such as coenzyme Q10 (3.6 wt\%; AQUA Q10 P40-NF, Nissin Pharmaceutical, Tokyo, Japan), niacin amid (0.7 wt\%), L-cystine (18.2 wt\%), ascorbic acid (34.2 wt\%), succinic acid (3.6 wt\%), fumaric acid (3.6 wt\%), Lglutamine (34.6 wt\%), and riboflavin (1.5 wt\%; Bislase inj, Toa Eiyo, Tokyo, Japan). TwX was given to the mice in TwX group by oral gavage once daily $20 \mathrm{mg} / \mathrm{kg}$ per day from $4.5 \mathrm{M}$ of age until sacrifice.

\section{Behavioral analysis}

Rotarod test was used to assess sensorimotor coordination of mice at ages of 2, 5, 7,9 and

$11 \mathrm{M}$, and evaluation criteria is the time that mice spent on the rotating rod (MK670; 
Muromachi Kikai Co., Tokyo, Japan) before falling as our previous report. ${ }^{11}$ In brief, the rotarod test began with mice trying to stay on a rod rotating at $4 \mathrm{rpm}$, the speed was then increased to $40 \mathrm{rpm}$ in a period of 60 seconds, and then kept the speed of $40 \mathrm{rpm}$. The latency to fall (up to a maximum of 400 seconds) was recorded. Six trials were performed in each measurement, and the best result was recorded.

8-arm radial maze test was performed when mice aged $3,6,8,10$ and $12 \mathrm{M}$ as our previous reports. ${ }^{8,12}$ Briefly, dietary restriction over 7 days was carried out which led to an $85 \%$ decrease of free-feeding body weight in all mice. Then mice were allowed to explore the baited arms of the maze for 10 min in 2 days habituation sessions. After adaptation, each mouse was left in the maze until either all pellets in 4 of the arms $(1,3,4$, and 7) were obtained or 5 min elapsed. Re-entry into the baited arms previously visited was recorded as a working memory error.

\section{Tissue preparation}

Mice aged $12 \mathrm{M}$ were deeply anesthetized and then perfused with $20 \mathrm{ml}$ chilled phosphatebuffered saline (PBS, pH 7.4), followed by 4\% paraformaldehyde (PFA) in PBS. After postfixed in 4\% PFA overnight, the brains were transferred into 10, 20 and 30\% (wt/vol) sucrose in PBS for $24 \mathrm{~h}$, respectively. Coronal brain sections $(20 \mu \mathrm{m})$ were cut on a cryostat at $-20^{\circ} \mathrm{C}$ and mounted on silane-coated glass slides.

\section{Histochemistry and immunohistochemistry}

For Nissl staining, brain sections were incubated in $0.1 \%$ cresyl violet (CV) for $5 \mathrm{~min}$ at room temperature, dehydrated gradually in ethanol, and coverslipped with microcoverglass. 
For single immunohistochemistry, after incubation in $0.3 \%$ hydrogen peroxide/methanol followed by $5 \%$ bovine serum albumin (BSA), the sections were strained overnight at $4^{\circ} \mathrm{C}$ with the following primary antibody: mouse anti-4G8 antibody (1:1000, Biolegend, San Diego, CA, USA), rabbit anti-A11 antibody (1:200, Invitrogen, Camarillo, CA, USA), goat anti-NLRP3 antibody (1:100, Abcam, Cambridge, MA, USA), mouse anti-caspase-1 antibody (1:200, Adipogen, San Diego, CA, USA), goat anti-IL-1 $\beta$ antibody (1:100, R\&D Systems, Minneapolis, MN, USA), rabbit anti-Iba-1 antibody (1:1000, Abcam), rabbit anti-TNF $\alpha$ antibody (1:200, Abcam), mouse anti-4-HNE antibody (1:40, JaICA, Shizuoka, Japan), mouse anti-8-OHdG antibody (1:20, JaICA) and rabbit anti- Nitrotyrosine antibody (1:200, Sigma-Aldrich, St. Louis, MO, USA). After washed with PBS, brain sections were treated with suitable biotinylated secondary antibodies (1:500; Vector Laboratories) at room temperature for $2 \mathrm{~h}$. Then the sections were incubated with the avidin-biotin-peroxidase complex (VECTASTAIN Elite ABC Kit; Vector Laboratories) for $30 \mathrm{~min}$ and visualized with 3,3'-diaminobenzidine (DAB). Negative control sections were stained in the same way as described above except for the primary antibodies.

3 sections per brain and 5 random selected regions were then analyzed with a light microscope (Olympus BX-51, Tokyo, Japan) for each measurement. Cerebral cortex (CTX), hippocampus (HI), and thalamus (TH) were measured for semiquantitative analysis of Nissl, A11, NLRP3, caspase-1, IL-1 $\beta$, Iba-1, TNF- $\alpha, 4-\mathrm{HNE}, 8-\mathrm{OHdG}$ and nitrotyrosine staining intensity. The number of 4G8-positive plaques was expressed per $1 \mathrm{~mm}^{2}$.

\section{Double immunofluorescent histochemistry}


After incubation in 5\% BSA in PBS with $0.1 \%$ triton at room temperature for $1 \mathrm{~h}$, the sections were incubated at $4{ }^{\circ} \mathrm{C}$ overnight with primary antibody. Antibodies were as follows: rabbit anti-A11 antibody (1:200, Invitrogen), goat anti-NLRP3 antibody (1:100, Abcam), mouse anti-Aß40 antibody (1:100, Wako, Osaka, Japan), and mouse anti- $\alpha$ SMA antibody (1:100, Sigma-Aldrich). Following washes, sections were incubated with fluorescent secondary antibody, and the fluorescent signals were visualized by confocal microscope (LSM-780; Zeiss, Jena, Germany).

\section{Statistical analysis}

Data are expressed as mean \pm standard deviation (SD). Statistical comparison was performed using one-way ANOVA analysis followed by Tukey-Kramer test. $p<0.05$ was considered significant.

\section{Results}

\section{Changes of $\mathrm{CBF}$ with ameroid constrictors implantation}

$\mathrm{CBF}$ gradually decreased in $\mathrm{CCH}$ and $\mathrm{CCH}+\mathrm{TwX}$ groups from $1 \mathrm{~d}$ after surgery, reached a minimum at $7 \mathrm{~d}$ and then became stable until $28 \mathrm{~d}$ (Fig. $1 \mathrm{~B},{ }^{\#} p<0.01$ vs APP23). There was no significant difference between $\mathrm{CCH}$ and TwX groups.

\section{Behavioral analysis after $\mathrm{CCH}$}

$\mathrm{CCH}$ and $\mathrm{CCH}+\mathrm{TwX}$ groups showed significant impairment of rotarod test at 5, 7,9 and $11 \mathrm{M}$ after surgery compared to WT and APP23 groups, and TwX treatment $(\mathrm{CCH}+\mathrm{TwX})$ 
significantly improved the rotarod time at $9 \mathrm{M}$ compared with $\mathrm{CCH}$ group (Fig. $1 \mathrm{C},{ }^{*} p<0.05$ and ${ }^{* *} p<0.01$ vs WT; ${ }^{\#} p<0.05$ and ${ }^{\# \#} p<0.01$ vs APP23; ${ }^{\$} p<0.05$ and ${ }^{\$ \$} p<0.01$ vs CCH). In 8-arm radial maze test, $\mathrm{CCH}+\mathrm{TwX}$ group showed significant improvement in errors at 8 , 10 and $12 \mathrm{M}$ compared with CCH group (Fig. $1 \mathrm{D},{ }^{*} p<0.05$ and ${ }^{* *} p<0.01$ vs WT; ${ }^{*} p<0.05$ vs APP $23 ;{ }^{\$} p<0.05$ and ${ }^{\$ \$} p<0.01$ vs $\left.\mathrm{CCH}\right)$.

\section{Neuronal loss after CCH}

Nissl staining showed significant lower density of neurons in subiculum, CA1, dentate gyrus (DG) and TH regions of 3 APP23 groups than in WT mice (Fig. 2A and 2B). However, TwX treatment significantly suppressed these neuropathological changes in the hippocampus and TH (Fig. 2B, ${ }^{* *} p<0.01$ vs WT; ${ }^{\#} p<0.05$ and ${ }^{\# \#} p<0.01$ vs APP $23 ;{ }^{\$} p<0.05$ and ${ }^{\$ \$} p<$ 0.01 vs $\mathrm{CCH})$.

\section{Parenchymal and vascular A $\beta$ deposits}

The numbers of 4G8 positive-amyloid plaque (Fig. 3A, arrows) and A11-positive amyloid oligomer formation (Fig. 3A) were increased in $\mathrm{CCH}$ group, which were reduced by $\mathrm{TwX}$ treatment (Fig. 3A, 3D and 3E, ${ }^{*} p<0.05$ and ${ }^{* *} p<0.01$ vs WT; ${ }^{\#} p<0.05$ and ${ }^{\# \#} p<0.01$ vs APP23; ${ }^{\$} p<0.05$ and ${ }^{\$ \$} p<0.01$ vs CCH).

Immunofluorescent analysis showed a colocalization of A $\beta$ oligomer with NLRP3 both in $\mathrm{A} \beta$ plaque and neural cell (Fig. 3B). A $\beta$ was also accumulated in cerebral vessels in $\mathrm{CCH}$ group than APP23 group, which was attenuated by TwX treatment (Fig. 3C). 


\section{Neuroinflammation after $\mathbf{C C H}$}

NLRP3, caspase-1 and IL-1 $\beta$ were evidently increased in 3 APP23 groups (Fig. 4A, 5A and 6A), and TwX treatment significantly reduced such enhanced expressions of NLRP3, caspase-1 and IL-1 $\beta$ compared with CCH group (Fig. 4B, 5B and 6B, ${ }^{*} p<0.05$ and ${ }^{* *} p<0.01$ vs WT; ${ }^{\#} p<0.05$ and ${ }^{\# \#} p<0.01$ vs APP23; ${ }^{\$} p<0.05$ and ${ }^{\$ \$} p<0.01$ vs CCH). The expression of the microglial Iba-1 was also increased in APP23 and APP23 $+\mathrm{CCH}$, especially in the cerebral cortex, but was inhibited by TwX treatment (Fig. 7A and 7B, ${ }^{*} p<0.05$ and ${ }^{* *} p<0.01$ vs WT; ${ }^{\#} p<0.05$ vs APP $23 ;{ }^{\$} p<0.05$ vs $\left.\mathrm{CCH}\right)$. The expression of pro-inflammatory cytokine marker TNF- $\alpha$ was increased significantly in the CTX, CA3 and TH, which was recovered by TwX treatment (Fig. 8A and 8B, ${ }^{*} p<0.05$ and ${ }^{* *} p<0.01$ vs WT; ${ }^{\#} p<0.05$ and ${ }^{\# \#} p<0.01$ vs APP $23 ;{ }^{\$} p<0.05$ and ${ }^{\$ \$} p<0.01$ vs $\left.\mathrm{CCH}\right)$.

\section{Oxidative stress change after $\mathrm{CCH}$}

As shown in Fig. 9-11, 4-HNE, 8-OHdG and nitrotyrosine were increased in CTX and HI in 3 APP23 groups (Fig. 9A, 10A and 11A). These oxidative stress markers were all attenuated in $\mathrm{CCH}+\mathrm{TwX}$ group compared with $\mathrm{CCH}$ group (Fig. 9B, 10B and 11B, ${ }^{*} p<0.05$ and ${ }^{* *} p<$ 0.01 vs WT; ${ }^{\#} p<0.05$ and ${ }^{\# \#} p<0.01$ vs APP $23 ;{ }^{\$} p<0.05$ and ${ }^{\$ \$} p<0.01$ vs CCH).

\section{Discussion}

The present study showed that the administration of antioxidative mixture TwX improved motor coordination and working memory (Fig. 1), rescued hippocampal neuron loss (Fig. 2), decreased $A \beta$ pathology (Fig. 3), and attenuated inflammatory reaction (Fig. 4-8) and oxidative 
stress (Fig. 9-11) under $\mathrm{CCH}$ of APP23 mice. $\mathrm{CCH}$ is one major cause of vascular dementia, but is also an important risk factor of $\mathrm{AD}$ progression. ${ }^{13}$ Our previous studies suggested that $\mathrm{CCH}$ enhanced neurodegenerative processes by promoting oxidative stress and neuroinflammation. ${ }^{7-8}$

Since hippocampal neurons are critically important for memory function, ${ }^{14}$ the positive effect on working memory (Fig. 1D) was most likely a result of the protective mechanism of TwX against $\mathrm{A} \beta$ pathology under $\mathrm{CCH}$ (Fig. 2). In the present study, TwX significantly reduced amyloid plaques, $\mathrm{A} \beta$ oligomer formation, and CAA in the brain (Fig. 3). We also observed an anti-inflammatory effect of TwX by reducing the expression of these inflammatory markers (Fig. 4-8). A $\beta$ oligomer induces the activation of the NLRP3 inflammasome, which then trigger downstream caspase- 1 , IL-1 $\beta,{ }^{15}$ microglia, ${ }^{2}$ and TNF- $\alpha .{ }^{16}$ Thus the present results revealed the suppression of $A \beta$ pathology based on the anti-inflammatory effects of TwX.

Oxidative stress is an early event in $\mathrm{AD}$, and also plays a key role in the progression of AD pathology. ${ }^{17-19}$ As was expected, TwX showed a positive effection on an early stage of A $\beta$ pathology with anti-oxidative mechanism in reducing the 4-HNE, 8-OHdG and nitrotyrosine (Fig. 9-11). Several studies have reported that antioxidants have potential therapeutic roles against $\mathrm{AD} .{ }^{20-22}$ Compared to a single ascorbic acid, the multiple antioxidant TwX showed synergistic effects on attenuating oxidative stress in an irradiation mouse model. ${ }^{23}$ Our previous study also showed the neuroprotective effects of $\mathrm{TwX}$ in an acute cerebral ischemia mouse model. ${ }^{9}$

In summary, the present study revealed that $\mathrm{CCH}$ enhanced primary $\mathrm{AD}$ pathology of APP23, and TwX improved motor and memory functions, reduced such primary AD pathology 
with suppressing inflammatory and oxidative stresses. 


\section{Acknowledgements}

This work was partly supported by a Grant-in-Aid for Scientific Research (B) 17H04196,

(C) 17K10827 and Challenging Research 15K15527, and by Grants-in-Aid from the Research

Committees (Mizusawa H, Nishizawa M, Sasaki H, and Aoki M) from the Ministry of Health,

Labour and Welfare of Japan. The authors wish to express their gratitude to Dr. Haruhiko Inufusa (TIMA Japan Corporation) for the kind gift of Twendee $\mathrm{X}$ used in this work.

\section{Conflict of interest}

The authors disclose no potential conflict of interests.

\section{References}

1. Hishikawa N, Fukui Y, Sato K, et al. Characteristic features of cognitive, affective and daily living functions of late-elderly dementia. Geriatr Gerontol Int 2016; 16: 458-465.

2. Selkoe DJ. Alzheimer's disease: genes, proteins, and therapy. Physiol Rev 2001; 81: 741-766.

3. Wang J, Gu BJ, Masters CL, Wang YJ. A systemic view of Alzheimer disease - insights from amyloid- $\beta$ metabolism beyond the brain. Nat Rev Neurol 2017; 13: 703.

4. Perry G, Nunomura A, Hirai K, et al. Oxidative damage in Alzheimer's disease: the metabolic dimension. Int J Dev Neurosci 2000; 18: 417-421.

5. Verdile G, Keane KN, Cruzat VF, et al. Inflammation and Oxidative Stress: The Molecular Connectivity between Insulin Resistance, Obesity, and Alzheimer's Disease. Mediators Inflamm 2015; 2015: 105828. 
6. Duncombe J, Kitamura A, Hase Y, et al. Chronic cerebral hypoperfusion: a key mechanism leading to vascular cognitive impairment and dementia. Closing the translational gap between rodent models and human vascular cognitive impairment and dementia. Clin Sci (Lond) 2017; 131: 2451-2468.

7. Shang J, Yamashita T, Zhai Y, et al. Strong Impact of Chronic Cerebral Hypoperfusion on Neurovascular Unit, Cerebrovascular Remodeling, and Neurovascular Trophic Coupling in Alzheimer's Disease Model Mouse. J Alzheimers Dis 2016; 52: 113-126.

8. Zhai Y, Yamashita T, Nakano Y, et al. Chronic Cerebral Hypoperfusion Accelerates Alzheimer's Disease Pathology with Cerebrovascular Remodeling in a Novel Mouse Model. J Alzheimers Dis 2016; 53: 893-905.

9. Kusaki M, Ohta Y, Inufusa H, et al. Neuroprotective Effects of a Novel Antioxidant Mixture Twendee X in Mouse Stroke Model. J Stroke Cerebrovasc Dis 2017; 26: 11911196.

10. Sturchler-Pierrat C, Abramowski D, Duke M, et al. Two amyloid precursor protein transgenic mouse models with Alzheimer disease-like pathology. Proc Natl Acad Sci U S A 1997; 94: 13287-13292.

11. Ohta $\mathrm{Y}$, Nagai M, Nagata $\mathrm{T}$, et al. Intrathecal injection of epidermal growth factor and fibroblast growth factor 2 promotes proliferation of neural precursor cells in the spinal cords of mice with mutant human SOD1 gene. J Neurosci Res 2006; 84: 980-992.

12. Kurata T, Miyazaki K, Kozuki M, et al. Atorvastatin and pitavastatin improve cognitive function and reduce senile plaque and phosphorylated tau in aged APP mice. Brain Res 2011; 1371: 161-170. 
13. Zhu X, Tian J, Sun S, et al. (-)-SCR1693 Protects against Memory Impairment and Hippocampal Damage in a Chronic Cerebral Hypoperfusion Rat Model. Sci Rep 2016; 6: 28908 .

14. Florian C, Roullet P. Hippocampal CA3-region is crucial for acquisition and memory consolidation in Morris water maze task in mice. Behav Brain Res 2004; 154: 365-374.

15. Heneka MT, Kummer MP, Stutz A, et al. NLRP3 is activated in Alzheimer's disease and contributes to pathology in APP/PS1 mice. Nature 2013; 493: 674-678.

16. Tobinick E, Gross H, Weinberger A, et al. TNF-alpha modulation for treatment of Alzheimer's disease: a 6-month pilot study. MedGenMed 2006; 8: 25.

17. Perry G, Cash AD, Smith MA. Alzheimer Disease and Oxidative Stress. J Biomed Biotechnol 2002; 2: 120-123.

18. Zhu X, Lee HG, Casadesus G, et al. Oxidative imbalance in Alzheimer's disease. Mol Neurobiol 2005; 31: 205-217.

19. Sultana R, Butterfield DA. Role of oxidative stress in the progression of Alzheimer's disease. J Alzheimers Dis 2010; 19: 341-353.

20. Harrison FE, Allard J, Bixler R, et al. Antioxidants and cognitive training interact to affect oxidative stress and memory in APP/PSEN1 mice. Nutr Neurosci 2009; 12: 203218

21. Dumont M, Kipiani K, Yu F, et al. Coenzyme Q10 decreases amyloid pathology and improves behavior in a transgenic mouse model of Alzheimer's disease. J Alzheimers Dis 2011; 27: 211-223.

22. Heo JH, Hyon-Lee, Lee KM. The possible role of antioxidant vitamin C in Alzheimer's 
disease treatment and prevention. Am J Alzheimers Dis Other Demen 2013; 28: 120125.

23. Inufusa H. Composition for protection against celldamaging effects, US Patent: 9089548 B2. issued date July 28th, 2015. 


\section{Figure Legends}

Fig. 1) Twendee $X(T w X)$ improved behavioral deficits of Alzheimer's disease (AD) mice model with chronic cerebral hypoperfusion $(\mathrm{CCH})$. (A) Surgical implantation of ameroid constrictors on bilateral common carotid arteries. (B) Temporal profile of the cerebral blood flow measured by laser-Doppler flowmeter. (C) Analysis of motor function by rotarod test, and (D) working memory by 8 -arm radial maze test $\left({ }^{* *} p<0.01\right.$ versus WT; ${ }^{\#} p<0.05$ versus APP23, ${ }^{\# \# p} p<0.01$ versus APP23; ${ }^{\$} p<0.05$ versus $\mathrm{APP} 23+\mathrm{CCH},{ }^{\$ \$} p<0.01$ versus APP $\left.23+\mathrm{CCH}\right)$.

Fig. 2) (A) Nissl stainings in cerebral cortex (CTX), hippocampus (HI), and thalamus (TH) of AD mice model with CCH. (B) Quantitative analysis of Nissl staining intensity in the CTX, subiculum (Sub), CA1, dentate gyrus (DG), and TH $\left({ }^{* *} p<0.01\right.$ versus WT; ${ }^{\#} p<0.05$ versus APP23, ${ }^{\#} p<0.01$ versus APP23; ${ }^{\$} p<0.05$ versus APP $23+\mathrm{CCH},{ }^{\$} p<0.01$ versus APP23+CCH. Scale bar $=50 \mu \mathrm{m})$.

Fig. 3) TwX ameliorated amyloid- $\beta$ (A $\beta$ ) pathology in $\mathrm{AD}$ mice model with $\mathrm{CCH}$. (A) 4G8 immunolabelling of A $\beta$ plaque (arrows) in CTX and HI, A11 immunolabelling of amyloid oligomer in CTX and TH. (B) Double immunofluorescence staining for A11 plus NLRP3 in A $\beta$ plaques and cells. (C) Double immunofluorescence staining for $\alpha$-SMA plus A $\beta 40$. (D) Quantitative analysis of amyloid plaques in CTX and HI, and (E) quantitative analysis of A11 staining intensity in CTX and TH $\left(\stackrel{*}{p}<0.05\right.$ versus $\mathrm{WT},{ }^{* *} p<0.01$ versus $\mathrm{WT} ;{ }^{\#} p<0.05$ versus APP23, ${ }^{\#} p<0.01$ versus APP $23 ;{ }^{\$} p<0.05$ versus APP $23+\mathrm{CCH},{ }^{\$} p<0.01$ versus APP $23+\mathrm{CCH}$. Scale bar $=50 \mu \mathrm{m})$. 
Fig. 4) Immunohistochemical staining of NLRP3 in AD mice model with CCH. (A) NLRP3 staining in CTX, HI and TH. (B) Quantitative analysis of NLRP3 $\left({ }^{*} p<0.05\right.$ versus $\mathrm{WT},{ }^{* *} p<$ 0.01 versus WT; ${ }^{\#} p<0.05$ versus APP23, ${ }^{\#} p<0.01$ versus APP23; ${ }^{\$} p<0.05$ versus $\mathrm{APP} 23+\mathrm{CCH},{ }^{\$} p<0.01$ versus APP $23+\mathrm{CCH}$. Scale bar $\left.=50 \mu \mathrm{m}\right)$.

Fig. 5) Immunohistochemical staining of caspase-1 in AD mice model with $\mathrm{CCH}$. (A) caspase1 staining in CTX, HI and TH. (B) Quantitative analysis of caspase- $1\left({ }^{*} p<0.05\right.$ versus WT, ${ }^{* *} p$ $<0.01$ versus WT; ${ }^{\#} p<0.05$ versus APP23; ${ }^{\$} p<0.05$ versus $\mathrm{APP} 23+\mathrm{CCH},{ }^{\$} p<0.01$ versus APP23 $+\mathrm{CCH}$. Scale bar $=50 \mu \mathrm{m})$.

Fig. 6) Immunohistochemical staining of IL-1 $\beta$ in AD mice model with $\mathrm{CCH}$. (A) IL-1 $\beta$ staining in CTX, HI and TH. (B) Quantitative analysis of IL-1 $\beta\left({ }^{*} p<0.05\right.$ versus WT, ${ }^{* *} p<$ 0.01 versus WT; ${ }^{\#} p<0.05$ versus APP23, ${ }^{\#} p<0.01$ versus APP23; ${ }^{\$} p<0.05$ versus APP $23+\mathrm{CCH},{ }^{\$} p<0.01$ versus APP23+CCH. Scale bar $\left.=50 \mu \mathrm{m}\right)$.

Fig. 7) Immunohistochemical staining of Iba- 1 in $A D$ mice model with $\mathrm{CCH}$. (A) Iba-1 staining in CTX, HI and TH. (B) Quantitative analysis of Iba-1 $\left({ }^{*} p<0.05\right.$ versus $\mathrm{WT},{ }^{* *} p<0.01$ versus WT; ${ }^{\#} p<0.05$ versus APP $23 ;{ }^{\$} p<0.05$ versus APP $23+\mathrm{CCH}$. Scale bar $\left.=50 \mu \mathrm{m}\right)$.

Fig. 8) Immunohistochemical staining of TNF- $\alpha$ in AD mice model with $\mathrm{CCH}$. (A) TNF- $\alpha$ staining in CTX, HI and TH. (B) Quantitative analysis of TNF- $\alpha\left({ }^{*} p<0.05\right.$ versus $\mathrm{WT},{ }^{* *} p<$ 
0.01 versus WT; ${ }^{\#} p<0.05$ versus $\mathrm{APP} 23,{ }^{\#} p<0.01$ versus $\mathrm{APP} 23 ;{ }^{\$} p<0.05$ versus $\mathrm{APP} 23+\mathrm{CCH},{ }^{\$} p<0.01$ versus APP23+CCH. Scale bar $\left.=50 \mu \mathrm{m}\right)$.

Fig. 9) Immunohistochemical staining of 4-HNE in AD mice model with $\mathrm{CCH}$. (A) 4-HNE staining in CTX, HI and TH. (B) Quantitative analysis of 4-HNE $\left({ }^{* *} p<0.01\right.$ versus WT; ${ }^{*} p<$ 0.05 versus APP $23 ;{ }^{\$} p<0.05$ versus APP $23+\mathrm{CCH},{ }^{\$} p<0.01$ versus APP23+CCH. Scale bar $=50 \mu \mathrm{m})$.

Fig. 10) Immunohistochemical staining of 8-OHdG in AD mice model with $\mathrm{CCH}$. (A) 8-OHdG staining in CTX, HI and TH. (B) Quantitative analysis of $8-\mathrm{OHdG}\left({ }^{*} p<0.05\right.$ versus $\mathrm{WT},{ }^{* *} p<$ 0.01 versus WT; ${ }^{\#} p<0.05$ versus $\mathrm{APP} 23,{ }^{\#} p<0.01$ versus $\mathrm{APP} 23 ;{ }^{\$} p<0.05$ versus $\mathrm{APP} 23+\mathrm{CCH},{ }^{\$} p<0.01$ versus APP23+CCH. Scale bar $\left.=50 \mu \mathrm{m}\right)$.

Fig. 11) Immunohistochemical staining of nitrotyrosine in $\mathrm{AD}$ mice model with $\mathrm{CCH}$. (A) Nitrotyrosine staining in CTX, HI and TH. (B) Quantitative analysis of nitrotyrosine $\left(^{*} p<0.05\right.$ versus WT, ${ }^{* *} p<0.01$ versus WT; ${ }^{\#} p<0.05$ versus APP23, ${ }^{\# \#} p<0.01$ versus APP23; ${ }^{\$} p<0.05$ versus APP23+CCH, ${ }^{\$} p<0.01$ versus APP23 $+\mathrm{CCH}$. Scale bar $\left.=50 \mu \mathrm{m}\right)$. 